\title{
On Fractional Differential Operators Involving Multivariable Generalized Hypergeometric Function
}

\author{
Ram Niwas Meghwal \\ Department of Mathematics, Government Bangur College, \\ Didwana (Nagaur) -341303 Rajasthan \\ meghwalramniwas@gmail.com
}

\begin{abstract}
In this paper we use fractional differential operators $D_{k, \alpha, x}^{n}$ and ${ }_{\alpha} D_{x}^{\mu}$ to derive a number of key formulas of multivariable H-function. We use the generalized Leibnitz's rule for fractional derivatives in order to obtain one of the aforementioned formulas, which involve a product of two multivariable's H-function. It is further shown that ,each of these formulas yield interesting new formulas for certain multivariable hyper geometric function such as generalized Lauricella function (Srivastava-Dauost)and Lauriella hyper geometric function some of these application of the key formulas provide potentially useful generalization of known result in the theory of fractional calculus.
\end{abstract}

Key words: Fractional differential operator, multivariable H-function.

AMS subject classification 2000 MSC: $26 a 3333 c 40$

\section{Introduction And Definitions}

The fractional derivative of special function of one and more variables is important such as in the evaluation of series, [10,15] the derivation of generating function [12,chap.5] and the solution of differential equations [4,14;chap-3] motivated by these and many other avenues of applications, the fractional differential operators $D_{k, \alpha, x}^{n}$ and ${ }_{\alpha} D_{x}^{\mu}$ are much used in the theory of special function of one and more variables .

We use the fractional derivative operator defined in the following manner [16]

$$
D_{k, \alpha, x}^{n}\left(x^{\mu}\right)=\prod_{r=0}^{n-1}\left[\frac{\sqrt{\mu+r k+1}}{\sqrt{\mu+r k-\alpha+1}}\right] \mathrm{x}^{\mu+n k}
$$

Where $\alpha \neq \mu+1$ and $\alpha$ and $k$ are not necessarily integers

We use the binomial expansion in the following manner

$$
\left(a x^{\mu}+b\right)^{\lambda}=b^{\lambda} \sum_{l=0}^{\infty}\left(\begin{array}{l}
\lambda \\
l
\end{array}\right)\left(\frac{a x^{\mu}}{b}\right)^{l} \quad \text { where }\left[\frac{a x^{\mu}}{b}\right]<1
$$

the familiar differential operator ${ }_{\alpha} D_{x}^{\mu}$ is defined by [5, p.49; 3; 9; 17, P-356]

$$
{ }_{\alpha} D_{x}^{\mu} f(\mathrm{x})=\left[\begin{array}{lc}
\frac{1}{\sqrt{-\mu}} \int_{\alpha}^{x}(x-t)^{-\mu-1} f(t) d t & , \quad[\operatorname{Re}(\mu)<0] \\
\frac{d^{m}}{d x^{m}}{ }_{\alpha} D_{x}^{\mu-m} f(x), & {[0 \leq \operatorname{Re}(\mu)<\mathrm{m}]}
\end{array}\right.
$$

Where $\mathrm{m}$ is a positive integer 
For $\alpha=0$,(1.3) Defines the classical Riemann-Liouville fractional derivative of order $\mu$ (or- $\mu$ ) when $\alpha \rightarrow \infty$ ( 1.3 ) may be identified with the definition of the well known Weyl fraction derivative of order $\mu$ (or- $\mu$ ) [1,chap.13);3] the special case of fractional calculus operator ${ }_{\alpha} D_{x}^{\mu}$ when $\alpha=0$ is written as $D_{x}^{\mu}$ thus we have

$$
D_{x}^{\mu}={ }_{0} D_{x}^{\mu}
$$

In this paper we drive several fractional derivative formulas involving multivariable $\mathrm{H}$-function which as defined by srivastav and panda [8, p.271 (4.1) et. Seq.] and studied systematically by then [6,7,8 also 11] for this multivariable H-function we adopt the contracted notations (due essentially to Srivastava and panda [13] thus following the various conventions and notations explained fairly and fully in their earlier works [6,7,8 see also 11,13]

$$
\left.H\left[z_{1}, \ldots, z_{r}\right]=H_{p, q: \mathrm{p}_{1}, q_{1}, \ldots, p_{r}, q_{r}}^{0, n: \mathrm{m}_{1} n_{1}, ., \mathrm{m}_{r}, n_{r}}\left[\begin{array}{c}
z_{1} \\
\vdots \\
z_{r}
\end{array}\right] \begin{array}{l}
\left(a_{j}, \alpha_{j}^{(1)} \ldots \alpha_{j}^{(r)}\right)_{1, p}:\left(c_{j}^{(1)}, \gamma_{j}^{(1)}\right)_{1, p 1}, \ldots,\left(c_{j}^{(r)}, \gamma_{j}^{(r)}\right)_{1, p_{r}} \\
\left(b_{j}, \beta_{j}^{(1)} \ldots \beta_{j}^{(r)}\right)_{1, q}:\left(d_{j}^{(1)}, \delta_{j}^{(1)}\right)_{1, q_{1}}, \ldots,\left(d_{j}^{r}, \delta_{j}^{(r)}\right)_{1, q_{r}}
\end{array}\right]
$$

Denote the H-function of r-variables $z_{1}, z_{2}, \ldots, z_{r}$ here for convenience

$\left(a_{j}, \alpha_{j}^{1}, \ldots \alpha_{j}^{(r)}\right)_{1, p} \quad$ Abbreviates the p- member array

$$
\left(a_{1}, \alpha_{1}^{1}, \ldots \alpha_{1}^{(r)}\right), \ldots,\left(a_{\mathrm{p}} \alpha_{p}^{1}, \ldots \alpha_{p}^{(r)}\right)
$$

parameters

$$
\left(\alpha_{j}^{(i)}, \gamma_{j}^{(i)}\right), \ldots \ldots \ldots \ldots \ldots,\left(\alpha_{p_{i}}^{(i)}, \gamma_{p_{i}}^{(i)}\right) \quad ; \quad(i=1, \ldots, r)
$$

and so on ,suppose , as usual that the parameters

$$
\begin{aligned}
& a_{j}, j=1, \ldots, p ; \quad c_{j}^{(i)}, j=1, \ldots, p_{i} ; \\
& b_{j}, j=1, \ldots, \mathrm{q} ; \quad d_{j}^{(i)}, j=1, \ldots, q_{i} ; \quad \forall i \in(i=1, \ldots, r)
\end{aligned}
$$

Are complex number and the associated coefficients

$$
\begin{array}{ll}
\alpha_{j,} j=1, \ldots, p ; \gamma_{j}^{(i)}, j=1 \ldots p_{i} ; & \\
\beta_{j}, j=1, \ldots, q ; \delta_{j}^{(i)}, j=1 . . . q_{i} ; & \forall i \in(1, \ldots, r)
\end{array}
$$

Are positive real numbers such that

$$
\Lambda_{i}=\sum_{j=1}^{r} \alpha_{j}^{(i)}-\sum_{j=1}^{q} \beta_{j}^{(i)}+\sum_{j=1}^{p_{i}} \gamma_{j}^{(i)}-\sum_{j=1}^{q_{i}} \delta_{j}^{(i)} \leq 0
$$


and

$$
\Omega_{i}=-\sum_{j=n+1}^{p} \alpha_{j}^{(i)}-\sum_{j=1}^{q} \beta_{j}^{(i)}+\sum_{j=1}^{n_{i}} \gamma_{j}^{(i)}-\sum_{j=n_{i}+1}^{p_{i}} \gamma_{j}^{(i)}+\sum_{j=1}^{m_{i}} \delta_{j}^{(i)}-\sum_{j=m_{2}+1}^{q_{i}} \delta_{j}^{(i)}>0 ; \quad \forall i \in[1, \ldots, r]
$$

Where the integers $\mathrm{n}, \mathrm{p}, \mathrm{q}, m_{i}, n_{i}, p_{i}, q_{i}$ are constrained by the inequalities $0 \leq n \leq p, \quad q \geq 0$, $1 \leq m_{i} \leq q_{i}, 0 \leq n_{i} \leq p_{i}[i=1, \ldots, r]$ and the equality $(1.10)$ holds true for suitably restricted values of the complex variables $z_{1}, \ldots ., z_{r}$

Then it is known that the multiple Mellin-Barnes counter integral [11, p.251 (c.1)] representing the multivariable H-function (1.5) converges absolutely under the condition (1.11) when

$$
\begin{gathered}
\left|\arg \left(z_{i}\right)\right|<\frac{1}{2} \Lambda \Omega_{i} \quad, \forall i \in[1, \ldots, r] \\
H\left[z_{1}, \ldots z_{r}\right]=\left|\begin{array}{cc}
0\left(\left|z_{1}\right|^{\xi_{1}} \ldots,\left|z_{r}\right|^{\xi_{r}}\right), & \left(\max \left|z_{1}\right|, \ldots,\left|z_{r}\right| \rightarrow 0\right) \\
0\left(\left|z_{1}\right|^{\eta_{1}} \ldots,\left|z_{r}\right|^{\eta_{r}}\right), & \left(\eta=0 ; \min \left|z_{1}\right|, \ldots,\left|z_{r}\right| \rightarrow \infty\right)
\end{array}\right|
\end{gathered}
$$

Where with $i=1, \ldots, r$

$$
\begin{aligned}
& \xi_{i}=\min \left\{\operatorname{Re}\left(d_{j}^{(i)} / \delta_{j}^{(i)}\right)\right\}, \quad\left(j=1, \ldots, m_{i}\right) \\
& \eta_{i}=\max \left\{\operatorname{Re}\left(c_{j}^{(i)}-1 / \gamma_{j}^{(i)}\right)\right\}, \quad\left(j=1, \ldots, n_{i}\right)
\end{aligned}
$$

Provided that each of the inequalities (1.10) (1.11) and (1.12) holds true.

Throughout the present paper .we assume that the convergence and existence condition corresponding appropriately to the ones detained above are satisfied by each of the various H-function involved in our results which are presented in the following sections

\section{Main Result}

In this section we shall prove our main formulas on fractional differential operator involving multivariable $\mathrm{H}$ function

\section{Result}

$$
\begin{aligned}
& D_{k, \alpha, x}^{n}\left\{x^{t}\left(x^{v_{1}}+a\right)^{\lambda}\left(b-x^{v_{2}}\right)^{-\delta}\right. \\
& \left.H\left[z_{1} x^{a}\left(x^{\nu_{1}}+a\right)^{\sigma_{1}}\left(b-x^{\nu_{2}}\right)^{-\delta}, \ldots, z_{r} x^{\rho_{r}}\left(x^{\mu_{1}}+a\right)^{\sigma_{r}}\left(b-x^{\nu_{2}}\right)^{-\delta_{\gamma}}\right]\right\}
\end{aligned}
$$

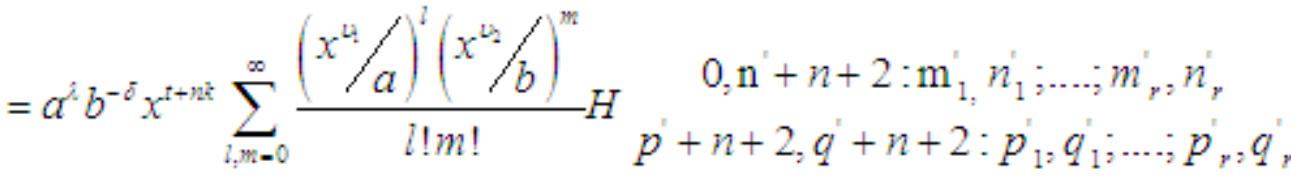




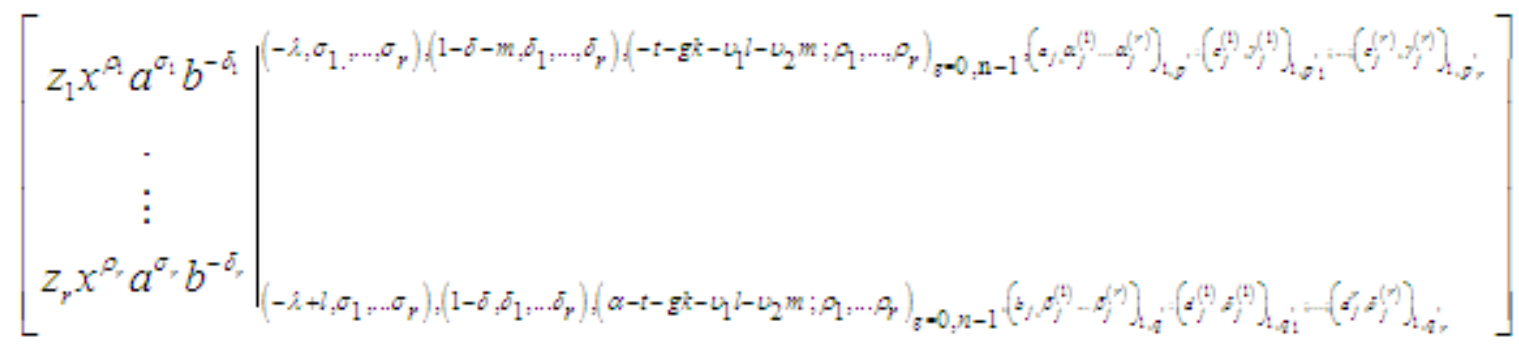

... (2.1)

Provided (in addition to the appropriate convergence and existence condition) that

$$
\begin{gathered}
\min \left\{v_{1}, v_{2}, \rho_{i}, \sigma_{i}, \delta_{i}\right\}>0 \quad(i=1, \ldots, r) ; \\
\max \left\{\left|\arg \left(x^{\nu_{1}} / a\right)\right|,\left|\arg \left(x^{\nu_{2}} / b\right)\right|\right\}<\pi \\
\operatorname{Re}(k)+\sum_{i=1}^{r} \rho_{i} \xi_{i}>-1
\end{gathered}
$$

Where $\xi_{i}=(i=1, \ldots ., r)$ are given in (1.14)

\section{Result}

$$
\begin{aligned}
& D_{k, a, z}^{*} D_{y}^{-}\left\{x^{4} y^{2}\left(x^{c_{4}}+a\right)^{2}\left(b-x^{v_{2}}\right)^{-b}\left(y^{b_{2}}+c\right)^{k}\left(d-y^{v_{s}}\right)^{-s}\right. \\
& H\left[z_{1} x^{a} y^{\lambda_{2}}\left(x^{v_{2}}+a\right)^{\sigma_{1}}\left(b-x^{v_{2}}\right)^{-b_{1}}\left(y^{v_{2}}+c\right)^{b_{a}}\left(d-y^{v_{s}}\right)^{-b_{2}}\right.
\end{aligned}
$$

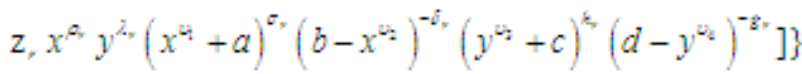

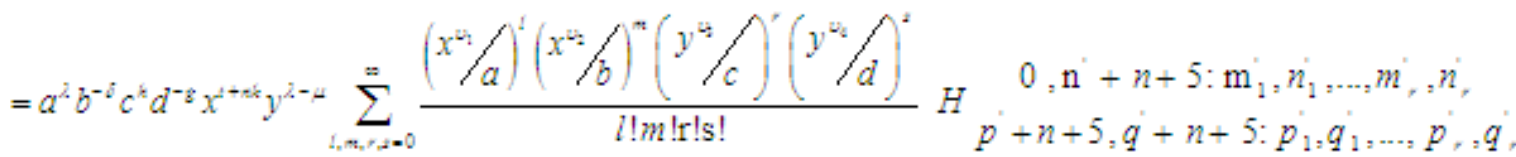

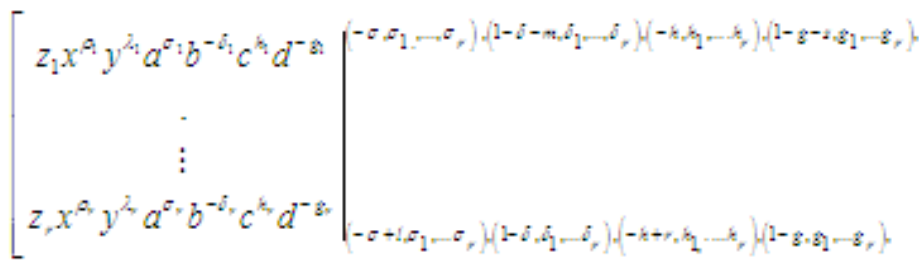

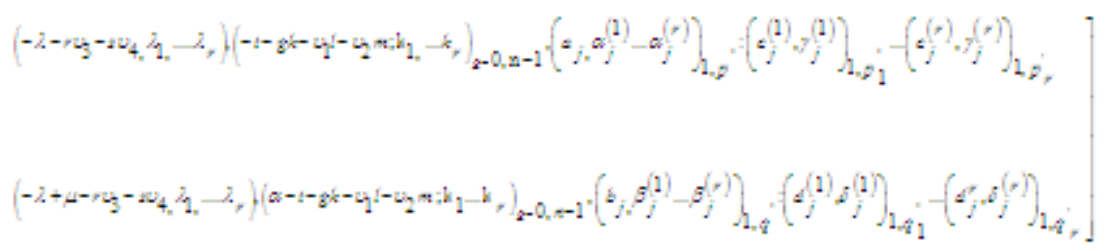

Provided (in addition to the appropriate convergence and existence conditions that

$$
\min \left(v_{1}, v_{2}, v_{3}, v_{4}, \sigma_{i}, \delta_{i}, h_{i}, g_{i}\right)>0(i=1, \ldots, \mathrm{r})
$$




$$
\begin{aligned}
& \max \left\{\left|\arg \left(x^{\nu_{1}} / a\right)\right|,\left|\arg \left(x^{\nu_{2}} / b\right)\right|, \arg \left|\left(y^{\nu_{3}} / c\right)\right|, \arg \left|\left(y^{\nu_{4}} / d\right)\right|\right\}<\pi \\
& \operatorname{Re}(k)+\sum_{i=1}^{r} k_{i} \xi_{i}>-1 \quad \operatorname{Re}(\lambda)+\sum_{i=1}^{r} \lambda_{i} \xi_{i}>-1
\end{aligned}
$$

And where $\xi_{1}, \ldots \xi_{r}$ are given in (1.14)

Proof of (2.1) :-

We first replace the multivariable $\mathrm{H}$-function occurring on the LHS by its Mellin Barnes contour integrals collected the powers of $\mathrm{x},\left(x^{\nu_{1}}+a\right)$ and $\left(b-x^{v_{2}}\right)$ and apply binomial expansion

$$
(x+\xi)^{\lambda}=\xi^{\lambda}\left(1+\frac{x}{\xi}\right)^{\lambda}=\xi^{\lambda} \sum_{l=0}^{\infty}\left(\begin{array}{l}
\lambda \\
l
\end{array}\right)\left(\frac{x}{\xi}\right)^{l} ; \quad\left|\frac{x}{\xi}\right|<1
$$

We then apply the fractional derivative operator in the following manner [2]

$$
D_{k, \alpha, x}^{n}\left(x^{\mu}\right)=\prod_{r=0}^{n-1}\left[\frac{\Gamma \mu+r k+1}{\Gamma \mu+r k-\alpha+1}\right] x^{\mu+n k}
$$

Where $\alpha \neq \mu+1$ and $\alpha$ and $\mathrm{k}$ are not necessarily integers and interpret the resulting Millen Barnes contour integrals as a H-function of r-variables we shall arrive at(2.1)

\section{Proof of (2.2):-}

We first replace the multivariable H-function occurring on the LHS by its Mellin- Barnes integrals

Collected the powers of $\mathrm{x}, \mathrm{y},\left(x^{\nu_{1}}+a\right),\left(b-x^{\nu_{2}}\right),\left(y^{v_{3}}+c\right),\left(d-y^{v_{4}}\right)$ and apply the binomial

$$
(x+\xi)^{\lambda}==\xi^{\lambda} \sum_{l=0}^{\infty}\left(\begin{array}{l}
\lambda \\
l
\end{array}\right)\left(\frac{x}{\xi}\right) ; \quad\left|\frac{x}{\xi}\right|<1
$$

We then apply the formula [7, p.67 eq.4.4.4]

$$
D_{x}^{\mu}\left(x^{\lambda}\right)=\frac{\Gamma 1+\lambda}{\Gamma 1+\lambda-\mu} x^{\lambda-\mu}, \quad[\operatorname{Re}(\lambda)>-1]
$$

and

$$
D_{k, \alpha, x}^{n}\left(x^{\mu}\right)=\prod_{r=0}^{n-1}\left[\frac{\Gamma \mu+r k+1}{\Gamma \mu+r k-\alpha+1}\right] x^{\mu+n k}
$$

Where $\alpha \neq \mu+1$ and $\alpha$ and $\mathrm{k}$ are not necessarily integers and interpret the resulting Mellin-Barnes contour integrals as a $\mathrm{H}$-function of r-variables we shall arrive at(2.2)

\section{Applications}

Each fractional derivative formula (2.1) and (2.2) has manifold generality. By specializing the various parameters and variables involved, these formulas (and indeed their several variations obtained by letting any desired number of the exponents: $\rho_{1}, \ldots, \rho_{r} ; \sigma_{1}, \ldots, \sigma_{r} ; \delta_{1}, \ldots, \delta_{r} ; \mathrm{h}_{1}, \ldots, h_{r} ; g_{1}, \ldots, g_{r} ; \lambda_{1}, \ldots, \lambda_{r}$ decrease to zero in such a manner that both the sides of the resulting equation exit) can suitably be applied to derive the corresponding results involving a wide variety of useful functions (or product of several such functions) which are expressible in terms of the E,F,G and $\mathrm{H}$-functions of one ,two and more variables. Say, if we put $\mathrm{n}=\mathrm{p}=\mathrm{q}=$ 0 ,the multivariable H-functions occurring on the LHS of (2.1) and (2.2) would immediately reduce to the product $\mathrm{r}$ (or s) different Fox's H- functions. Various special cases of Fox's H-function can be seen in a monograph of Mathai and Saxena [18, p.145-159].Thus it can also easily be derived fractional derivative formulas involving any of these simpler special functions desired. 
(1) Replacing $\delta$ by $(-\delta)$ in (2.1) and (2.2) ; setting $\sigma_{i}, \delta_{i} \rightarrow 0(i=1, \ldots ., r)$ in (2.1) and (2.2), in addition to these we replace $\mathrm{g}$ by $(-g)$ also set $h_{i}, g_{i} \rightarrow 0(i=1, \ldots, r)$ we get the following more elegant formulas :

$$
\begin{aligned}
& D_{k, \alpha, x}^{n}\left\{x^{t}\left(x^{v_{1}}+a\right)^{\lambda}\left(b-x^{v_{2}}\right)^{-\delta} H\left[z_{1} x^{\rho_{1}}, \ldots, \mathrm{z}_{r} x^{\rho_{r}}\right]\right\} \\
& =a^{\lambda} b^{-\delta} x^{t+n k} \sum_{l, m=0}^{\infty}\left(\begin{array}{l}
\lambda \\
l
\end{array}\right)\left(\begin{array}{l}
\delta \\
m
\end{array}\right) \frac{\left(x^{\nu_{1}} / a\right)^{l}\left(x^{v_{2}} / b\right)^{m}}{l ! m !} \\
& H \quad 0, \mathrm{n}^{\prime}+n: \mathrm{m}_{1,}^{\prime} n_{1}^{\prime} ; \ldots ; m_{r}^{\prime}, n_{r}^{\prime} \\
& p^{\prime}+n, q^{\prime}+n: p_{1}^{\prime}, q_{1}^{\prime} ; \ldots ; p_{r}^{\prime}, q_{r}^{\prime} \\
& {\left[\left.\begin{array}{c}
z_{1} x^{\rho_{1}} \\
\cdot \\
\cdot \\
z_{r} x^{\rho_{r}}
\end{array}\right|_{\left(\alpha-t-k g-v_{1} l-v_{2} m ; \rho_{1}, \ldots, \rho_{r}\right)_{g=0, n-1},\left(b_{j,}, \beta_{j}^{(1)} \ldots \beta_{j}^{(r)}\right)_{1, q^{\prime}}:\left(d_{j}^{(1)}, \delta_{j}^{(1)}\right)_{1, q_{1}^{\prime} ; \ldots ;\left(d_{j}^{r}, \delta_{j}^{(r)}\right)_{1, q_{r}^{\prime}}}} ^{\left(-t-k g-v_{1} l-v_{2} m ; \rho_{1}, \ldots, \rho_{r}\right)_{g=0, \mathrm{n}-1},\left(a_{j}, \alpha_{j}^{(1)} \ldots \alpha_{j}^{(r)}\right)_{1, p^{\prime}}:\left(c_{j}^{(1)}, \gamma_{j}^{(1)}\right)_{1, p_{1}^{\prime}, \ldots ;\left(c_{j}^{(r)}, \gamma_{j}^{(r)}\right)_{1, p_{r}^{\prime}}}}\right]}
\end{aligned}
$$

$$
\begin{aligned}
& D_{k, \alpha, x}^{n} D_{y}^{\mu}\left\{x^{t} y^{\lambda}\left(x^{\nu_{1}}+a\right)^{\lambda}\left(b-x^{v_{2}}\right)^{-\delta}\left(y^{v_{3}}+c\right)^{h}\left(d-y^{v_{4}}\right)^{-g}\right. \\
& \left.H\left[z_{1} x^{k_{1}} y^{\lambda_{1}}, \ldots \ldots, \mathrm{z}_{r} x^{\rho_{r}} y^{\lambda_{r}}\right]\right\} \\
& =a^{\lambda} b^{-\delta} c^{h} d^{-g} x^{t+n k} y^{\lambda-\mu} \sum_{l, m, r, s=0}^{\infty}\left(\begin{array}{l}
\sigma \\
l
\end{array}\right)\left(\begin{array}{l}
\delta \\
m
\end{array}\right)\left(\begin{array}{l}
h \\
r
\end{array}\right)\left(\begin{array}{l}
g \\
s
\end{array}\right) \frac{\left(x^{\nu_{1}} / a\right)^{l}\left(x^{\nu_{2}} / b\right)^{m}\left(y^{v_{3}} / c\right)^{r}\left(y^{v_{4}} / d\right)^{s}}{l ! m ! \mathrm{r} ! \mathrm{s} !} \\
& H \quad 0, \mathrm{n}^{\prime}+n+1: \mathrm{m}_{1}^{\prime}, n_{1}^{\prime}, \ldots, m_{r}^{\prime}, n_{r}^{\prime} \\
& p^{\prime}+n+1, q^{\prime}+n+1: p_{1}^{\prime}, q_{1}^{\prime}, \ldots, p_{r}^{\prime}, q_{r}^{\prime}
\end{aligned}
$$

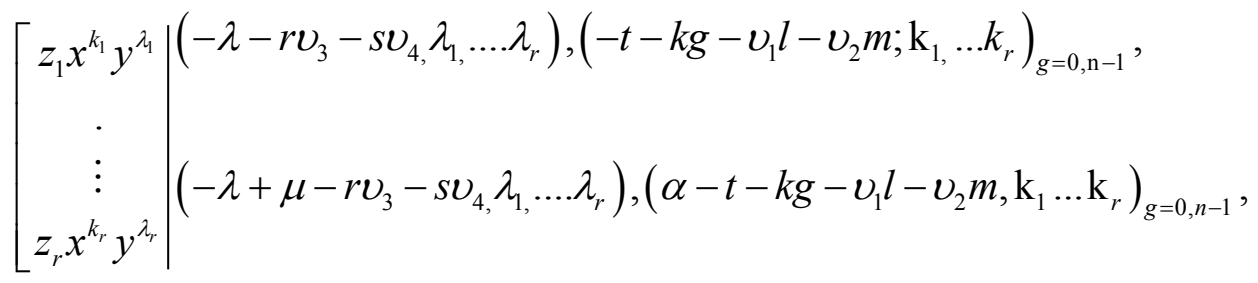

$$
\begin{aligned}
& \left.\left(a_{j}, \alpha_{j}^{(1)} \ldots \alpha_{j}^{(r)}\right)_{1, p^{\prime}}:\left(c_{j}^{(1)}, \gamma_{j}^{(1)}\right)_{1, p_{1}^{\prime}} \ldots\left(c_{j}^{(r)}, \gamma_{j}^{(r)}\right)_{1, p_{r}^{\prime}}\right] \\
& \left.\left(b_{j}, \beta_{j}^{(1)} \ldots \beta_{j}^{(r)}\right)_{1, q^{\prime}}:\left(d_{j}^{(1)}, \delta_{j}^{(1)}\right)_{1, q_{1}^{\prime}} \ldots\left(d_{j}^{r}, \delta_{j}^{(r)}\right)_{1, q_{r}^{\prime}}\right]
\end{aligned}
$$




\section{Acknowledgement}

The author is highly grateful toDr. B. S. Sinsinwar and Dr. Atul Garg for Their valuable help and suggestions to improve this paper

\section{References}

[1]. Eredlyi A. et.al: Tables of Integral Transform, vol.2 Mc Graw Hill ,NY/Toronto/London (1954)

[2]. Lavoie J. L, Osler and Tremblay R: Fractional Derivatives and Special Functions SIAM, Rev.18(1976),240-268

[3]. Samko, S.G. Kilbas,A. A. and Maricev,O.L.: Integrals and Derivatives of Fractional order some of their Applications,Nauka,Tekhnika Minsk(1987) in Russian

[4]. Nishimoto,K: Fractional Calculus Vol.1-4 Descrates Press ,Koriyama ,(1984,1987,1989, and 1991)

[5]. Oldham K.B.and Spanier.J: The Fractional Calculus, Acadmic Press NY/London,(1974)

[6]. Srivastava, H.M. and Panda R: Some expansion theorems and generating relations for the H-Function of several Complex variable I and II, Comment.math, univ.st.paul, 24. (1975) Fasc.2, 119-137; ibid25 (1976), Fasc.2, 167-197

[7]. Srivastava H.M. and Panda R. Some expansion theorem for the H-Function of several complex variables J.Reine Angew Math.288(1976),129-145

[8]. Srivastava, H.M. and Panda R: Some bilateral generations Function for a class of generalized hypergeometric polynomials ,J.Reine Angew Math. 283-284 (1976),265-274

[9]. Srivastava, H.M. and Panda R:Some multiple Integral Transformations involving the H-Function of several

[10]. variables Nederl,Acad.wetensch proc. Ser. A 82=Indag Math.41(1979) 353-36210. Srivastava ,H.M and Panda R: Certain Multidimensional Integral Transformations I and II Neder .Acad.Wetensch proc.ser. indag.math40(1980),118-131 and 132-144

[11]. Srivastava ,H.M.Gupta K.C. and Goyal S.P. :The H-Function of one and two variables with application South Asian Publicatios New Delhi/Madrs(1982)

[12]. Srivastava, H.M and Monocha,H.L.: A Treatise on Generating Functions Halsted Press Chichester and wiley, NY/Chichester/Brisbane/Toronto(1984)

[13]. Srivastava, H.M and Goyal S.P.: Fractional Derivatives of the H-Function of several variables J.Math.Anal Appl. 112,2(1985),641 651

[14]. Srivastava, H.M andBuschman R.G.: Theory and Application of Convolution Integral equation ,Kluwer ,Dordrecht Boston/London,(1992)

[15]. Srivastava R., Some application of fractional calculus in univalent functions , fractional calculus and their (H.M.Srivastava and S.Owa Eda) 371-382 Halsted Press Chichester and wiley, NY/Chichester/ Brisbane/Toronto(1989)

[16]. Misra A.P. Ganita,26(1975),1-18

[17]. Watanable, Y.: Notes on the Generalized Derivative of Riemann-Liouville and its applications to Leibnitz's formula I and II ,Tonoku, Math. 34 (1931), 8-37 and 28-41

[18]. Mathai,A.M. and Saxena ,R. K. : The H-function with application in statistics and other disciplines, Halsted press (Wiley eastern Ltd. ,New Delhi/ Banglore / Bombay) Wiley ,N Y / London/Sydney/Toronto,(1970). 